\title{
Magnetic Flux Density Feedback Control for Permanent Magnetic- Electromagnetic Hybrid Suspension System
}

\author{
Qiang CHEN, Jie LI, Guanchun LI, Siyang ZHOU \\ College of Mechatronics Engineering and Automation, National University of Defense Technology, 410073 Changsha, China
}

\begin{abstract}
Permanent magnetic-electromagnetic hybrid suspension system can effectively reduce energy consumption and heat release of the system, but also increase the difficulty of suspension control because of the existence of permanent magnets. The traditional current feedback control method is not conducive to the stability of the system and is difficult to debug. In this paper, the models of permanent magnetic-electromagnetic hybrid suspension system based on current feedback and magnetic flux density feedback are established. The effects of current feedback and magnetic flux density feedback on the stability of the system are analyzed in theory and the advantages of flux density feedback are pointed out. The model of magnet flux feedback is simple and it can overcome the disadvantages of current feedback, which is beneficial to the stability of the system. The magnetic flux density feedback control of permanent magnetic-electromagnetic hybrid suspension system is realized by simulation and experiment. Control system performs well and is easy to debug.
\end{abstract}

\section{Introduction}

Maglev transportation has advantages of safe, comfort, low noise, small turning radius and strong climbing ability [1]. It is a promising way of rail transit, which has received extensive attention in recent years. Countries such as Germany, Japan, China, South Korea and America have carried out relevant research on maglev technology and all have made great progress $[2,3]$. In permanent magnetic-electromagnetic (PM-EM) hybrid suspension system [4], the permanent magnets provide static suspension force, which electromagnets provide dynamic electromagnetic force required to adjust. It also has the advantages of low energy consumption, low heat release and small installed capacity of vehicle power. While at the same time, the difficulty of the suspension control becomes harder because of the existence of permanent magnets.

Suspension control is the core technique of PM-EM hybrid suspension system. Currently the major method of suspension control is gap-current double loops cascade control [5]. The gap outer-loop realizes stable suspension of the system and tracks the change of the gap. The current inner-loop realizes the quick response of the system. However, for the PM-EM hybrid suspension system, the relationship of magnetic force, suspension gap and current is complex and overlapping. The interloop using current feedback control is not beneficial to the design of suspension controller and the development of suspension performance. Considering that for the PMEM hybrid suspension system, the magnetic force is decided by the magnetic flux density in the suspension gap only, therefore using magnetic flux density feedback control inter-loop can make the design of the suspension controller easier. Besides, it will be more convenient to debug the control system [6, 7].

In this paper, relevant theoretical research is carried out. By comparing with the current feedback control, the effects of current feedback and magnetic flux density feedback on the stability of the system are analyzed and the advantages of magnetic flux density feedback control are pointed out. Using the magnetic flux density feedback control method realized the PM-EM hybrid suspension system stable suspension in simulation and experiment.

\section{System modelling}

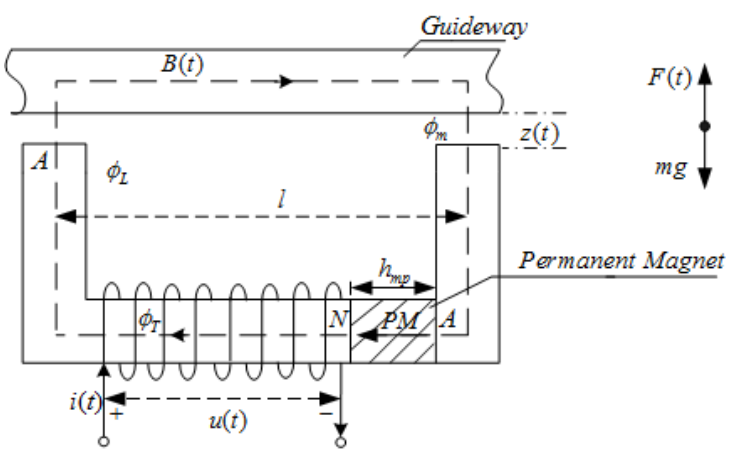

Figure 1. The Schematic diagram of single point model of PMEM hybrid suspension system.

The PM-EM hybrid suspension system is made up of permanent magnets and electronic magnets. In PM-EM 
hybrid suspension system, each bogie has two suspension modules. Each module has two suspension points. By mechanical and electrical decoupling, each suspension point is controlled separately. Thus the hybrid suspension system can be decomposed and considered as single point suspension system. Single point model of permanent magnetic-electromagnetic (PM-EM) hybrid suspension system is shown in Fig. 1.

The following assumptions are made for the single point suspension system:

(1) The leakage flux of coil winding, permanent magnet and the magnetic saturation characteristics of materials are ignored.

(2) The reluctance of magnet in core and guide rail is ignored, which means that the magnetic potential drops on the permanent magnet and the gap uniformly.

(3) The elastic vibration or dynamic deformation of the track is not considered, which means that the related stiffness coefficient of the track and the electronic magnet is infinite.

Considering the gap and the current as state variables, the single point model of the PM-EM hybrid suspension system can be described as

$$
\left\{\begin{array}{c}
m \ddot{z}(t)=-F(t)+m g \\
F(t)=\frac{\mu_{m p} \mu_{r} A\left[N i(t)+H_{c} h_{m p}\right]^{2}}{\left[2 \mu_{r} z(t)+h_{m p}\right]^{2}} \\
u(t)=R i(t)+\frac{\mu_{m p} A N^{2}}{2 \mu_{r} z(t)+h_{m p}} \dot{i}(t)-\frac{2 N A \mu_{m p} \mu_{r}\left[N i(t)+H_{c} h_{m p}\right]}{\left[2 \mu_{r} z(t)+h_{m p}\right]^{2}} \dot{z}(t)
\end{array}\right.
$$

Considering the gap and the magnetic flux density as the state variables, the single point model of the PM-EM hybrid suspension system can be described as

$$
\left\{\begin{array}{c}
m \ddot{z}(t)=-F(t)+m g \\
F(t)=\frac{A}{\mu_{0}} B^{2}(t) \\
u(t)=\frac{R}{\mu_{m p} N}\left\{\left[2 \mu_{r} z(t)+h_{m p}\right] B(t)-\mu_{m p} H_{c} h_{m p}\right\}+N A \dot{B}(t)
\end{array}\right.
$$

The meanings of symbols are illustrated in table 1 .

Table 1. Meanings of symbols for the PM-EM hybrid suspension system.

\begin{tabular}{|c|c|}
\hline Symbol & Meaning \\
\hline$\phi_{m}$ & Gap flux \\
\hline$\phi_{T}$ & Main pole flux \\
\hline$\phi_{L}$ & Leakage flux \\
\hline$B(t)$ & magnetic flux density \\
\hline$u(t)$ & Electromagnet coil voltage \\
\hline$i(t)$ & Electromagnet coil current \\
\hline$R$ & Electromagnet coil resistance \\
\hline
\end{tabular}

\begin{tabular}{|c|c|}
\hline$N$ & Electromagnet coil turns \\
\hline$A$ & cross-sectional area of iron core \\
\hline$H_{c}$ & Coercive force of permanent magnet \\
\hline$h_{m p}$ & Permanent magnet thickness \\
\hline$\mu_{0}$ & Air permeability \\
\hline$\mu_{m p}$ & Permeability of permanent magnet \\
\hline$\mu_{r}$ & Relative permeability of permanent magnet \\
\hline$z(t)$ & Gap between magnet and track \\
\hline$F(t)$ & electromagnetic force \\
\hline$m$ & The total mass \\
\hline$g$ & Gravitational acceleration \\
\hline
\end{tabular}

The magnetic flux density in the suspension gap of the PM-EM hybrid suspension system can be expressed as

$$
B(t)=\frac{\mu_{m p}\left[N i(t)+H_{c} h_{m p}\right]}{2 \mu_{r} z(t)+h_{m p}}
$$

Therefore formula 1 and formula 2 are equal, and it can be easily proved that the PM-EM hybrid suspension system is a non-linear and unstable system. But if current is defined as the state variable, from formula 1 , the magnetic force is correlated with many variables such as current and gaps, and the change of current will cause the change of gaps. The relationship of them is complex and coupling. However, using magnet flux density as state variable, from formula 2 , it can be seen that magnetic force is directly related with magnet flux density. The equation is simple. Therefore, using the magnetic flux density feedback inter-loop control, the suspension controller is easier to design and debug.

\section{Comparison of magnetic flux density feedback and current feedback}

\subsection{Current feedback}

Linearizing formula 1 at the equilibrium point $\left(z_{0}, i_{0}\right)$

$$
\left\{\begin{array}{c}
\Delta \ddot{z}=-\frac{1}{m} \Delta F \\
\Delta F=\frac{2 N A \mu_{m p} \mu_{r}\left(N i_{0}+H_{c} h_{m p}\right)}{\left(2 \mu_{r} z_{0}+h_{m p}\right)^{2}} \Delta i-\frac{4 A \mu_{m p} \mu_{r}^{2}\left(N i_{0}+H_{c} h_{m p}\right)^{2}}{\left(2 \mu_{r} z_{0}+h_{m p}\right)^{3}} \Delta z \\
\Delta u=R \Delta i+\frac{\mu_{m p} A N^{2}}{2 \mu_{r} z_{0}+h_{m p}} \Delta \dot{i}-\frac{2 N A \mu_{m p} \mu_{r}\left(N i_{0}+H_{c} h_{m p}\right)}{\left(2 \mu_{r} z_{0}+h_{m p}\right)^{2}} \Delta \dot{z}
\end{array}\right.
$$

Let 


$$
\begin{gathered}
K_{I Z}=\frac{2 N A \mu_{m p} \mu_{r}\left(N i_{0}+H_{c} h_{m p}\right)}{\left(2 \mu_{r} z_{0}+h_{m p}\right)^{2}} \\
K_{Z Z}=\frac{4 A \mu_{m p} \mu_{r}^{2}\left(N i_{0}+H_{c} h_{m p}\right)^{2}}{\left(2 \mu_{r} z_{0}+h_{m p}\right)^{3}} \\
K_{U I}=\frac{2 \mu_{r} z_{0}+h_{m p}}{\mu_{m p} A N^{2}} \\
K_{Z I}=\frac{2 \mu_{r}\left(N i_{0}+H_{c} h_{m p}\right)}{\left(2 \mu_{r} z_{0}+h_{m p}\right) N}
\end{gathered}
$$

Making $(\Delta z, \Delta \dot{z}, \Delta i)^{T}$ as state variable, the state-space equation is

$$
\left[\begin{array}{c}
\Delta \dot{z} \\
\Delta \ddot{z} \\
\Delta \dot{i}
\end{array}\right]=\left[\begin{array}{ccc}
0 & 1 & 0 \\
\frac{1}{m} K_{Z Z} & 0 & -\frac{1}{m} K_{I Z} \\
0 & K_{Z I} & -R K_{U I}
\end{array}\right]\left[\begin{array}{c}
\Delta z \\
\Delta \dot{z} \\
\Delta i
\end{array}\right]+\left[\begin{array}{c}
0 \\
0 \\
K_{U I}
\end{array}\right] \Delta u
$$

The block diagram of PM-EM hybrid suspension control system based on current feedback inter-loop is shown in Fig. 2. Using gap-current double loops cascade control. Gap outer-loop is gap feedback controller $C_{z}(s)$, which realizes the system levitate stably and track the change of gaps. Current inter-loop is current feedback controller $C_{i}(s)$, which makes the system response quickly.

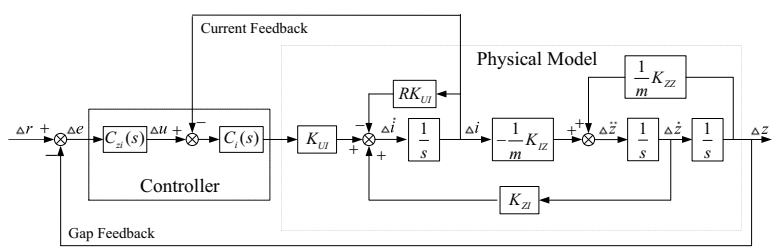

Figure 2. The block diagram of PM-EM hybrid suspension control system based on current feedback inter-loop.

The transfer function of current loop is

$$
G_{i}(s)=\frac{\Delta i(s)}{\Delta u(s)}=\frac{K_{U I} C_{i}(s)}{s+R K_{U I}+K_{U I} C_{i}(s)}
$$

The current loop uses proportional control, $C_{i}(s)=k_{i}$ and the current loop is a first order system. Time constant is

$$
\tau_{i}=\frac{1}{R K_{U I}+K_{U I} k_{i}}
$$

If there is no current inter-loop, the transfer function from control voltage $\Delta u$ to electromagnetic current $\Delta i$ is

$$
G_{i}^{\prime}(s)=\frac{K_{U I}}{s+R K_{U I}}
$$

Time constant will be

$$
\tau_{i}^{\prime}=\frac{1}{R K_{U I}}
$$

So current feedback makes the time constant of current loop smaller, and the current of electromagnets can be adjusted to track the change of control voltage in very short time, the response velocity will be quicker.

In order to investigate the effect of current feedback on the whole system, do not consider the gap feedback at first and the transfer function from $\Delta u$ to $\Delta z$ is

$$
\frac{\Delta z(s)}{\Delta u(s)}=-\frac{k_{i} K_{U I} K_{I Z}}{m s^{3}+\left(k_{i}+R\right) K_{U I} m s^{2}+\left(K_{I Z} K_{Z I}-K_{Z Z}\right) s-\left(k_{i}+R\right) K_{U I} K_{Z Z}}
$$

The root locus of the transfer function 14 with the change of current feedback coefficient $k_{i}$ is shown in Fig. 3 . With the increase of $k_{i}$, the unstable pole in the right half s-plane will increase, making the system diverge faster. So current feedback cannot solve the stable problem, nor it will make the suspension system more unstable.

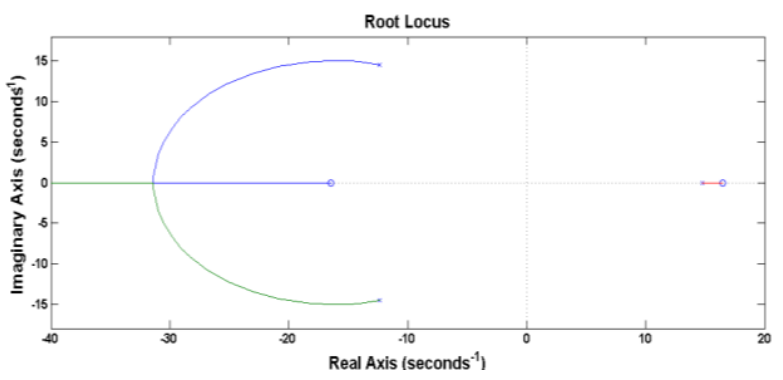

Figure 3. The root locus of current feedback control.

\subsection{Magnet flux density feedback}

Linearizing formula 2 at equilibrium point $\left(z_{0}, B_{0}\right)$.

$$
\left\{\begin{array}{c}
\Delta \ddot{z}(t)=-\frac{1}{m} \Delta F \\
\Delta F=\frac{2 A B_{0}}{\mu_{0}} \Delta B \\
\Delta u=N A \Delta \dot{B}+\frac{\left(2 \mu_{r} z_{0}+h_{m p}\right) R}{\mu_{m p} N} \Delta B+\frac{2 \mu_{r} R B_{0}}{\mu_{m p} N} \Delta z
\end{array}\right.
$$

Let

$$
\begin{gathered}
K_{B Z}=\frac{2 A B_{0}}{\mu_{0}} \\
K_{B B}=\frac{\left(2 \mu_{r} z_{0}+h_{m p}\right) R}{\mu_{m p} A N^{2}} \\
K_{Z B}=\frac{2 \mu_{r} R B_{0}}{\mu_{m p} A N^{2}}
\end{gathered}
$$

Making $(\Delta z, \Delta \dot{z}, \Delta B)^{T}$ as state variable, the state-space equation is 


$$
\left[\begin{array}{c}
\Delta \dot{z} \\
\Delta \ddot{z} \\
\Delta \dot{B}
\end{array}\right]=\left[\begin{array}{ccc}
0 & 1 & 0 \\
0 & 0 & -\frac{1}{m} K_{B Z} \\
-K_{Z B} & 0 & -K_{B B}
\end{array}\right]\left[\begin{array}{c}
\Delta z \\
\Delta \dot{z} \\
\Delta B
\end{array}\right]+\left[\begin{array}{c}
0 \\
0 \\
\frac{1}{N A}
\end{array}\right] \Delta u
$$

The block diagram of the PM-EM hybrid suspension control based on the feedback inter-loop of the magnetic flux density is shown in Fig. 4. Using gap-magnet flux density double loops cascade control. Gap outer-loop control realizes the system levitate stably and track the change of the gap. The magnet flux density inter-loop realizes the quick response of the system.

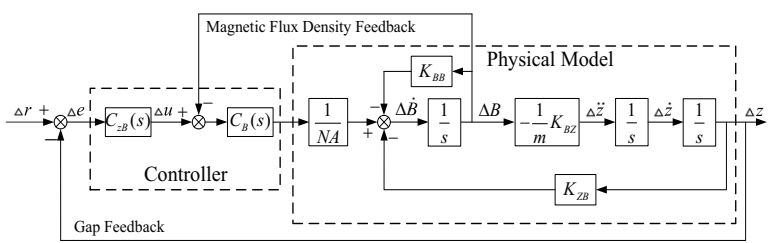

Figure 4. The block diagram of the PM-EM hybrid suspension control based on magnetic flux density feedback inter-loop.

The transfer function of magnet flux density loops is

$$
G_{B}(s)=\frac{\Delta B(s)}{\Delta u(s)}=\frac{C_{B}(s)}{N A s+N A K_{B B}+C_{B}(s)}
$$

The magnet flux density inter-loop uses proportional control, $C_{B}(s)=k_{B}$, and the magnet flux density loop is first order system. Time constant is

$$
\tau_{B}=\frac{N A}{N A K_{B B}+k_{B}}
$$

If there is no magnet flux density inter-loop, the transfer function from control voltage $\Delta u$ to gap magnet flux density $\Delta B$ is

$$
G_{B}^{\prime}(s)=\frac{1}{N A s+N A K_{B B}}
$$

Time constant will be

$$
\tau_{B}^{\prime}=\frac{1}{K_{B B}}
$$

So magnet flux density feedback makes the time constant of magnet flux density loop smaller, and the magnet flux density can be adjusted to track the change of control voltage in very short time, the velocity of the response will be quicker. At the same time, it is conducive to system control because magnetic force is decided by magnet flux density directly.

In order to investigate the effect of magnet flux density feedback on the whole system, do not consider the gap feedback at first and the transfer function from $\Delta u$ to $\Delta z$ is

$$
\frac{\Delta z(s)}{\Delta u(s)}=-\frac{k_{B} K_{B Z}}{m N A s^{3}+\left(k_{B}+N A K_{B B}\right) m s^{2}-N A K_{B Z} K_{Z B}}
$$

The root locus of the transfer function 24 with the change of magnet flux density feedback coefficient $k_{B}$ is shown in Fig. 5. With the increase of $k_{B}$, the unstable pole $p$ goes near the virtual axis. While $k_{B} \rightarrow \infty, p=0$, the system will be critical stable. It can be seen that the magnet flux density feedback is beneficial to the stabilization of the system.

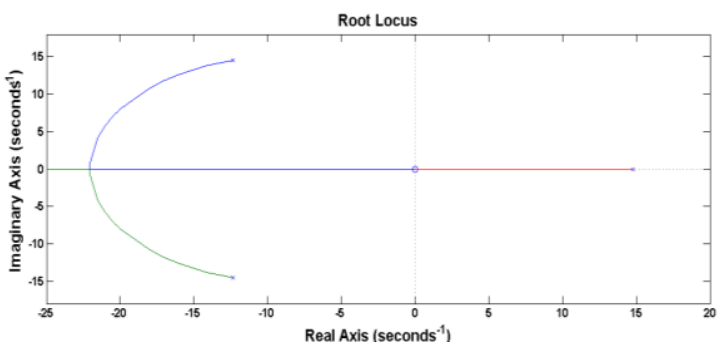

Figure 5. The root locus of magnet flux density feedback.

\section{Simulation and experiment}

Adapting the control system in Fig. 4, proportional control is used in magnet flux density inter-loop. $C_{B}(s)=k_{B}$, PID controller is used in the gap outer-loop. $C_{z i}(s)=k_{z i p}+k_{z i p} \frac{1}{s}+k_{z i d} s$, the air gap is $9 \mathrm{~mm}$, tracking amplitude $1 \mathrm{~mm}$ square wave. The change of the gap in simulation is shown in Fig. 6.

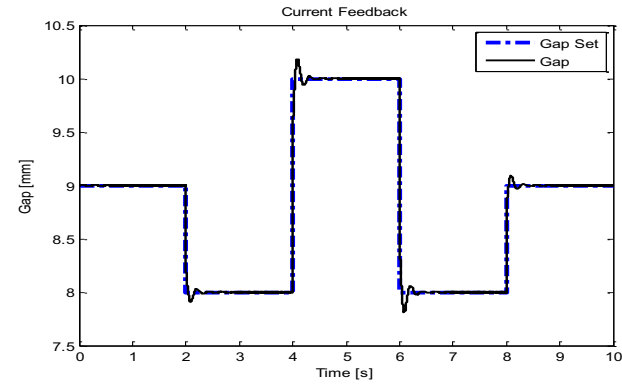

Figure 6. simulation results of tracking square wave.

Conducting suspension control experiment on the single bogie experimental platform shown in Fig. 7, using the same control method mentioned above. The air gap is $9 \mathrm{~mm}$, tracking amplitude $1 \mathrm{~mm}$ square wave. The change of the gap is shown in Fig. 8. Magnet flux density curve is shown in Fig. 9.

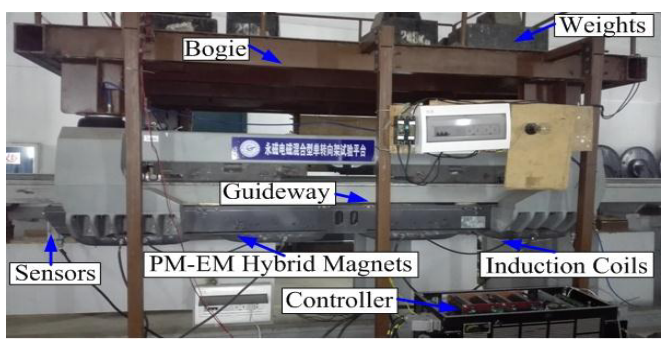

Figure 7. Single bogie experimental platform of the PM-EM hybrid suspension system. 


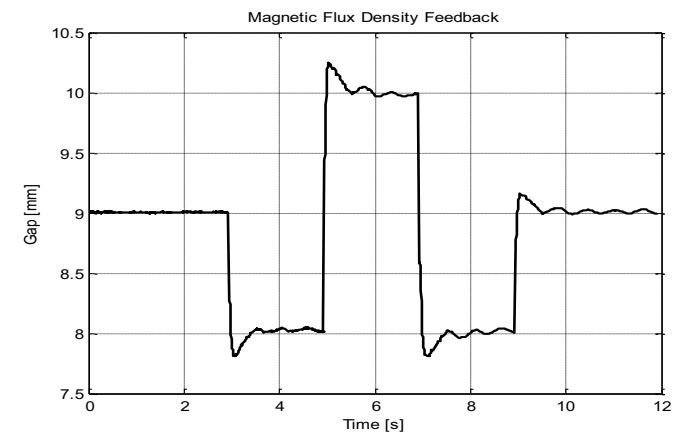

Figure 8. Experiment results of tracking square wave.

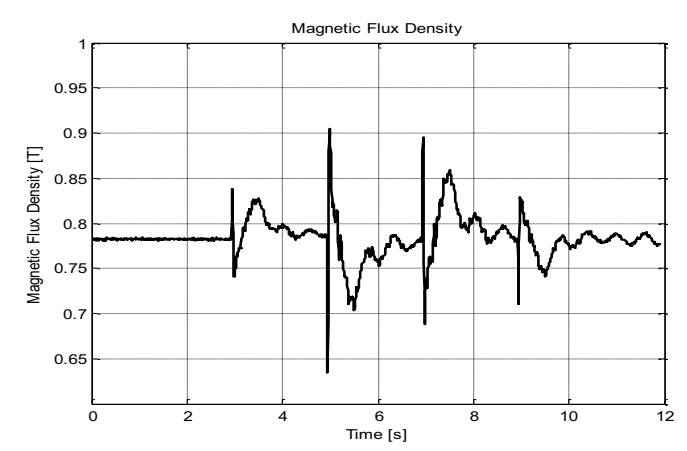

Figure 9. Magnet flux density curve.

From the simulation and experiment, because of the effect of gap outer-loops, the hybrid suspension system can be stable and achieve good control performance. However, the method of magnet flux density feedback is easier and is convenient to debug.

\section{Conclusion}

The influence of the current feedback inter-loop and the magnet flux feedback inter-loop on the PM-EM hybrid suspension control system is analyzed in theory based on the system model. Current feedback inter-loop is not conducive to the stability of the system and the performance of the outer-loop will be affected, what's worse, the system may be diverged. The model of magnet flux feedback is simple and it can overcome the disadvantage of current feedback, which is beneficial to the stability of the system. The results of the simulation and experiment show that the magnet flux feedback control system performs well, and it is easy to debug.

\section{References}

1. Lee H W, Kim K C, and Lee J. Review of Maglev Train Technologies. IEEE Transactions on Magnetics, 2006, 42(7):1917 1925.

2. Yan L G. Development and Application of the maglev transportation System. IEEE Transactions on Applied Superconductivity, 2008, 18(2):92-99.

3. Yan L G. Progress of the Maglev Transportation in China. IEEE Transactions on Applied Superconductivity, 2006, 16(2):1138 1141.

4. Cho H W, Han H S, Lee J M, Kim B S, Sung S Y. Design Considerations of EM-PM Hybrid Suspension and Propulsion Device for Magnetically Levitated Vehicle. IEEE Transactions on Magnetics, 2009, 45(10): 4632 4635.

5. LI Jin-hui, Zhou Dan-feng, LI Jie, Zhang Geng, Modeling and simulation of CMS04 Maglev Train with Active Controler, Journal of Central South University of Technology, 2013, 4 (22) : 13661377.

6. Wenqing Zhang, Jie Li, Kun Zhang, Peng Cui. Measurement and Control of Magnetic Flux Signal in Maglev System. Asian Journal of Control, 2014, 16(6), 1-11.

7. Wenqing Zhang, Jie Li, Kun Zhang, Peng Cui. Design of Magnetic Flux Feedback Controller in Hybrid Suspension System. Mathematical Problems in Engineering, 2013. 